\title{
Ethnologie de la Prise en Charge Alimentaire de l'enfant Allaité en Communauté Baoulé
}

\section{ESJ Humanities}

\section{Koffi N'Dri Célestin}

Socio-anthropologue de la Santé, Université Alassane Ouattara de Bouaké, Côte d'Ivoire

\section{Ouattara Zié Adama}

Socio-anthropologue de la Santé, $\mathrm{PhD}$, Chercheur Associé au Centre de Recherche pour le Développement (CRD), Université Alassane Ouattara de Bouaké, Côte d'Ivoire

\section{Brou Kouamé Aristide}

Doctorant en Sociologie et Anthropologie de la Santé à l'Université Alassane Ouattara de Bouaké, Chercheur Associé au Centre Suisse de Recherche Scientifique (CSRS); Côte d'Ivoire

\section{Abe N'Doumy Noel}

Anthropologue et Sociologue de la Santé, Professeur des Universités, Université Alassane Ouattara de Bouaké, Côte d'Ivoire

Submitted: 08 February 2020

Accepted: 04 December 2020

Published: 31 December 2020

Corresponding author:

N'dri Celestin Koffi

DOI: 10.19044/esj.2020.v16n35p172

(c)

Copyright 2020 Koffi N'Dri Célestin1 et. At. Distributed under Creative Commons BY-NC-ND 4.0 OPEN ACCESS

\section{Résumé}

Le présent article a pour objectif général d'analyser la prise en charge de l'enfant allaité chez les communautés Baoulé. De façon spécifique, il permet de comprendre l'alimentation de l'enfant allaité chez les Baoulé d'une part et d'autre part, les habitudes alimentaires de la nourrice dans la prise en charge de l'enfant allaité. Cette étude s'est déroulée en 2016 auprès des sous-groupes Baoulé, Faly de Bouaké et Nananfouè de Yamoussoukro. Les enquêtées étaient composées de femmes de troisième âge et de matrones, auprès desquelles ont été réalisés douze entretiens semi structurés individuels et quatre focus groups. Les entretiens individuels étaient adressés aux matrones et les focus groups animés avec les femmes de troisième âge. $\mathrm{Au}$ total, quatre focus groups et douze entretiens individuels ont été réalisés. Le nombre de femmes de troisième âge ayant participé aux focus groups est 32 , soit 8 femmes par focus group et 12 matrones ont été interviewées individuellement. Les entretiens ont été enregistrés à l'aide d'un dictaphone et transcrits entièrement puis les données ont été analysées. L'analyse globale des données a été obtenue par synthèse transversale des analyses des entretiens individuels et des focus groups. Pour des raisons éthiques, les identités des répondantes ont été remplacées par des prénoms fictifs. L'étude a révélé d'une part que la prise en charge alimentaire de l'enfant allaité est fondée sur l'alimentation. Il s'agit du lait maternel, des compléments alimentaires et de la période de sevrage. D'autre part, elle a montré que la nourrice doit se passer des aliments comme la mangue, le foutou couché, l'œuf, l'attiéké, le silure et le maïs frais pour une bonne santé de l'enfant allaité. Ainsi, le respect et l'application de ces pratiques et connaissances 
socioculturelles seraient une garantie à l'amélioration de la santé de l'enfant allaité. En somme, la santé de l'enfant allaité découle d'un suivi minutieux de son alimentation et de l'alimentation de la nourrice. Car, l'alimentation serait fondamentale pour la santé de l'enfant allaité.

Mots clés: ethnologie, prise en charge alimentaire, enfant allaité, baoulé

\title{
Ethnology of the Nutritional Care of the Child Breastfed in Baoulé Community
}

\author{
Koffi N'Dri Célestin \\ Socio-anthropologue de la Santé, \\ Université Alassane Ouattara de Bouaké, Côte d'Ivoire
}

Ouattara Zié Adama,

Socio-anthropologue de la Santé, $\mathrm{PhD}$, Chercheur Associé au Centre de Recherche pour le Développement (CRD), Université Alassane Ouattara de Bouaké, Côte d'Ivoire

\section{Brou Kouamé Aristide,}

Doctorant en Sociologie et Anthropologie de la Santé à l'Université Alassane

Ouattara de Bouaké, Chercheur Associé au Centre Suisse de Recherche

Scientifique (CSRS); Côte d'Ivoire Abe N'Doumy Noel,

Anthropologue et Sociologue de la Santé, Professeur des Universités, Université Alassane Ouattara de Bouaké, Côte d'Ivoire

Abstract

This paper focuses on analyzing the management of breastfed children in Baoulé communities. It provides an understanding of the feeding of breastfed children among the Baoulé as well as the nurse's feeding habits for the care of breastfed children. The study highlights the need to take into account the socio-cultural knowledge in the care of breastfed children in Baoulé communities. It was also conducted in 2016 among the Baoulé, Faly of Bouaké, and Nananfouè of Yamoussoukro subgroups. The respondents were composed of elderly women and matrons, which included twelve semistructured individual interviews and four focus groups. The individual interviews were addressed to the matrons and the focus groups were conducted with senior women. A total of four focus groups and twelve individual interviews were conducted. The number of elderly women who participated in the focus groups was 32, i.e., 8 women per focus group and 12 matrons were interviewed individually. The interviews were recorded using a dictaphone 
and transcribed in full, and the data were analyzed. The overall data analysis was obtained by cross-cutting synthesis of the analysis of the individual interviews and focus groups. For ethical reasons, the respondents' identities were replaced by fictitious first names. The study revealed, on the one hand, that the nutritional management of the breastfed child is based on feeding. This includes breast milk, food supplements, and the weaning period. On the other hand, it was shown that the nurse must do without foods such as mangoes, couch potato, egg, attiéké, sheatfish, and fresh corn for the good health of the breastfed child. The respect and application of these socio-cultural practices and knowledge would be a guarantee for the improvement of the health of the breastfed child. In short, the health of the breastfed child is the result of careful monitoring of his or her diet and of the nurse's feeding. Nutrition is therefore fundamental to the health of the breastfed child.

Keywords: Ethnology, alimentary care, breastfed child, baoulé

\section{Introduction}

La santé du binôme mère-nouveau-né est suivi dès le premier jour de l'accouchement. Les jours et les semaines qui suivent la naissance appelés période postnatale, représentent une phase cruciale dans la vie d'une mère et de son nouveau-né.

Deborah Lupton (2013), présente cela comme deux personnes inséparables et une complicité se crée entre la mère et l'enfant. Elle qualifie cette relation d'intercorporéité. «La notion d'intercorporéité pour comprendre la manière dont les mères «pensent et ressentent le corps de leurs enfants ». L'intercorporéité implique que des corps «apparemment autonomes et individualisés sont en fait expérimentés à un niveau phénoménologique comme entremêlés ». [...] D'une part, comme c'était le cas in utero, le corps de l'enfant se développe littéralement à partir de celui de la mère. D'autre part, les mères peuvent ressentir cette fusion à un niveau émotionnel : allaiter, ce serait en quelque sorte accepter de continuer à "partager" son corps pendant plusieurs mois, voire plusieurs années après l'accouchement ».

Kouakou (2014), mentionne dans son étude que l'allaitement immédiat après l'accouchement est faiblement pratiqué en Côte d'Ivoire, soit une proportion de $9 \%$. Les résultats de ses recherches montrent également que la connaissance de la recommandation d'initier l'allaitement maternel dans les trente premières minutes après la naissance selon le protocole national n'est pas du tout suivie car seulement $9 \%$ des femmes allaitantes ont dit avoir pratiqué la mise au sein immédiate.

Dans les communautés Baoulé étudiées, cette vulnérabilité du binôme mère-enfant et du nouveau-né de façon particulière est perçue, et mobilise un ensemble de pratiques de prise en charge endogènes. Cette étude se propose 
d'analyser la prise en charge de l'enfant allaité chez les communautés Baoulé. De façon spécifique, elle permet de comprendre l'alimentation de l'enfant allaité chez les Baoulé d'une part et d'autre part, les habitudes alimentaires de la nourrice dans la prise en charge de l'enfant allaité.

Ce travail s'inscrit dans la perspective de la théorie des pratiques. Cette théorie propose de situer les structures cognitives et symboliques directement au cœur des pratiques sociales nécessitant de placer ces dernières au centre de l'analyse. Autrement, une "pratique » est comme un type de comportement routinier qui consiste en plusieurs éléments interconnectés entre eux : des formes d'activités corporelles, des formes d'activités mentales, des « choses » et leur usage, des connaissances de base constituées de compréhension, savoirfaire, états émotionnels et motivations, selon (Reckwitz, 2002). Dans le cadre de cette recherche, cette théorie est utilisée pour montrer l'importance de l'usage des pratiques communautaires dans la prise en charge alimentaire de l'enfant allaité.

\section{Méthode et matériels}

Cette étude a été menée, auprès de sous-groupes Baoulé de la souspréfecture de Bouaké et de Yamoussoukro. Dans la sous-préfecture de Bouaké, l'étude a été réalisée auprès des Baoulé Faly dans le village de Bamoro. Quant à la sous-préfecture de Yamoussoukro, il s'agit des Baoulé Nananfouè dans le village de N'Zéré. Lors de la collecte des données de thèse, nous avons assisté à quelques séances de Communication pour le Changement de Comportement (CCC) de la sage-femme auprès des nourrices dans le village de Bamoro. Au cours de cette communication, la sage-femme sensibilisait les nourrices sur les types d'allaitement, les périodes d'allaitement, comment allaiter etc. En dépit de cela, les nourrices en communauté alimentent l'enfant selon les principes communautaires, sans toutefois tenir compte des instructions de la sage-femme. Au regard de ce comportement, nous avons décidé de nous imprégner du mode d'alimentation de l'enfant allaité dans la communauté. Le village N'Zéré était le village de la pré-enquête pour la thèse. Ce qui pourrait expliquer le choix de ces sites.

Les données ont été collectées en 2016 selon une approche qualitative mobilisant des techniques, notamment l'entretien semi structuré et le focus group. Les informations ont été collectées auprès de deux différents groupes de femmes. Il s'agit des femmes du troisième âge et des matrones.

Dans les communautés étudiées, les personnes en charge des conseils aux jeunes filles, femmes procréatrices sont des matrones et des femmes de troisième âge. Ces dernières sont sollicitées, pour la plupart, quand les femmes sont enceintes. Elles interviennent pour le suivi de la grossesse jusqu'à l'accouchement. Ces matrones étant moins nombreuses dans les communautés d'étude, à partir de la technique de boule de neige, nous les avons enquêtées 
jusqu'à atteindre un seuil de saturation. Pour une question d'éthique, les identités affectées aux enquêtées dans le texte sont des prénoms fictifs.

$\mathrm{Au}$ total, quatre focus groups et douze entretiens individuels ont été réalisés. Le nombre de femmes de troisième âge ayant participé aux focus groups est 32, soit 8 femmes par focus group et 12 matrones ont été interviewées individuellement. De manière détaillée, 16 femmes de troisième âge et 6 matrones ont été enquêtées dans chacun des deux villages. Les entretiens ont été enregistrés à l'aide d'un dictaphone et transcrits entièrement puis les données ont été analysées. L'analyse globale des entretiens a été obtenue par synthèse transversale des analyses des entretiens individuels et des focus groups.

\section{Résultats}

La prise en charge alimentaire de l'enfant allaité en communauté Baoulé est axée sur l'alimentation de façon générale.

\section{L'alimentation de l'enfant allaité}

L'alimentation de l'enfant allaité dans la communauté est multiforme et prend en compte l'allaitement maternel, l'aliment de complément et la période de sevrage.

\section{1-1.1 L'allaitement maternel}

Les groupes culturels Baoulé perçoivent le lait maternel comme la nourriture principale de l'enfant allaité. Il assure le développement, la croissance et permet à l'enfant allaité d'être en embonpoint. L'enfant allaité est nourri régulièrement pour préserver sa santé. En dépit de cela, le lait maternel a selon les communautés étudiées, des effets néfastes sur certaines parties du corps de l'enfant allaité. Pendant l'allaitement, si le lait maternel tombe sur le sexe masculin de l'enfant allaité, cela pourrait rendre l'enfant allaité infertile plus tard. Pour pallier cela, au cours de l'allaitement la mère doit avoir une position adéquate ; c'est-à-dire être bien assise avant d'allaiter son enfant. En outre, elle doit se rassurer que le sexe de l'enfant allaité est bien protégé. Une matrone de 67 ans interviewée, dit à cet effet ce qui suit :

"Chez nous, avant de donner sein à l'enfant, la mère doit s'asseoir, la nuit comme la journée. Parce que le lait de sein c'est dangereux, ça gâte beaucoup de chose sur l'enfant. Lorsque la mère donne le sein à l'enfant, si le lait verse sur le sexe masculin de l'enfant ça le rend impuissant. Donc une mère doit faire beaucoup attention quand elle donne sein à son enfant garçon ». 
L'analyse synthétique des focus groups (100\%) et des entretiens individuels (75\%) montre que dans la communauté Baoulé, il y a des prescriptions sociales à respecter. En effet, il est déconseillé d'allaiter l'enfant lorsque la mère est longuement exposée au soleil. Sous l'effet des rayons du soleil, le lait maternel change de température. Il est imprégné de chaleur et considéré comme étant «chaud». Car, ce lait qualifié de «chaud» provoquerait la diarrhée ou le vomissement chez l'enfant allaité. Les rayons du soleil sont donc perçus comme un facteur pathogène, qui affecte la qualité du lait maternel. Les propos ci-après étayent ce qui précède :

"Chez nous ici, quand tu vas travailler dans soleil tout ton corps est chaud (dégager une chaleur). Quand c'est comme ça, si tu donnes sein à l'enfant (faire téter l'enfant allaité), ce n'est pas bon; parce que, quand sein-là est chaud que tu donnes à l'enfant ça donne diarrhée. Quand c'est comme ça que tu veux donner sein à l'enfant, tu attends d'abord; et quand ton corps n'est plus chaud, là si tu donnes sein n'y a pas de problème », extrait d'un focus group avec femme du troisième âge.

"Chez nous les Baoulé, quand on travaille dans soleil, on ne donne pas sein directement à l'enfant; parce que ce n'est pas bon, quand sein est chaud que tu donnes à l'enfant, ça le fait vomir. Quand on travaille au soleil avant de donner sein à l'enfant on se repose d'abord, là il n'y a pas de problème », extrait de l'entretien semi directif avec la matrone Modjou 58 ans.

Outre, l'allaitement maternel, dans l'alimentation de l'enfant allaité chez les Baoulés enquêtés, l'on note le recours à des aliments de complément.

\section{1-2 L'aliment de complément}

Les aliments de complément donnés aux enfants en état d'allaitement, sont composés de la bouillie de céréales et/ou du plat familial. En ce qui concerne des céréales, il s'agit, de la bouillie de farine de maïs, mil, et riz. Pour obtenir la bouillie, les céréales sont d'abord moulues avant de procéder à la cuisson. Quant à la cuisson, la quantité de farine est fonction de la quantité d'eau prise. La cuisson peut durer 25-30 minutes. Lorsque la bouillie se refroidit, la mère nourrit l'enfant allaité à l'aide d'une cuillère à café, après avoir ajouté deux ou trois morceaux de sucre. Selon les perceptions des enquêtées, les farines de maïs et de mil peuvent être conservées sur une période de deux semaines, contrairement à la farine de riz qui ne fait qu'une semaine. Ces farines sont conservées dans des récipients avec des fermetures. Pour ce qui est du plat familial, il s'agit, de l'igname et du manioc. L'igname est donnée en purée avec des ajouts de poisson maquereau, d'l'huile végétale 
comestible et du sel. La mère se sert également de la cuillère à café ou l'index pour le lui donner à manger. Pour le manioc, la pâte de manioc est pilée à l'aide d'un mortier et d'un pilon, pour la rendre compacte. À la pâte obtenue, on y ajoute de l'eau pour qu'elle soit liquide. Ce liquide est tamisé à l'aide d'une passoire. Une fois cela est fait, le liquide reste décanter durant 30 minutes, le temps que l'eau remonte à la surface et stagne. Cette eau est retirée, et par la suite, la pâte homogène est mise au feu à travers une casserole ou marmite. Pendant la cuisson, la mère fait usage d'une spatule en bois pour malaxer. Le temps qu'il faut pour la cuisson est environ 1 heure 30 minutes. Après la cuisson, on obtient ce qu'on appelle le 'placali', qui se consomme avec une sauce faite à base de gombo mélangé et de jus de graine de palme. Difficile à mâcher, ce type de sauce a pour avantage de faire glisser les bouchées facilement. Ces différents mets sont considérés comme les plats de résistance de l'enfant allaité. Lorsque l'enfant en état d'allaitement est nourri avec ces aliments, cela permet à la mère de pouvoir vaquer à ses occupations, dans la mesure où cela réduit considérablement la fréquence d'allaitement. Selon ces communautés, lorsque l'enfant allaité consomme ces aliments, il dort pendant longtemps, il ne pleure pas et la mère parvient à faire ses activités. Pour la plupart, l'enfant allaité consomme ces aliments à partir du quatrième mois. D'après ces communautés, à cet âge le lait maternel ne peut rassasier l'enfant en état d'allaitement. Les focus groups mettent en évidence ces modes d'alimentation de l'enfant allaité au sein des communautés. C'est ce qui transparait de cet extrait d'un focus group avec les femmes du troisième âge :

"Chez nous, quand l'enfant est encore nouveau-né, quand on donne sein fini, on donne aussi de l'eau à boire, quand c'est comme ça là il est bien rassasié, il ne pleure pas et il dort bien. Quand c'est comme ça tu arrives à travailler, à faire tes petits trucs. Au moment, il commence à grandir un peu : quand il a trois (3) ou quatre (4) mois, on donne baka (bouillie de farine) de riz, de mil et maïs. Mais, maïs là on n'aime pas trop donner, parce que souvent ça donne mal de ventre à l'enfant. Quand l'enfant mange ça là, il ne te fatigue pas, tu peux faire ton travail, ça le fait grossir aussi ».

Dans la communauté, une mère nourrit son enfant en état d'allaitement au regard de ses faits et gestes. Souvent, l'enfant allaité saisi le bras de sa mère quand elle mange ou il fixe sa mère lorsqu'elle met la nourriture à la bouche. Quand elle ne lui en donne pas, il pleure. Au regard de cette attitude, elle déduit que l'enfant allaité est intéressé par la nourriture familiale. Ce qui expliquerait l'introduction précoce de l'aliment de complément. Cette expression de l'appétit chez l'enfant allaité est traduite par les focus groups comme le montre l'extrait suivant : 
"Ici, souvent quand l'enfant à quatre ou cinq mois, quand sa maman mange il la regarde. Quand la mère envoie la main vers la bouche il la regarde, quand elle envoie la main vers la nourriture il la regarde encore; et il fait même chose. Parfois, l'enfant attrape bras de sa maman (prendre la main de sa mère), ou il pleure. Quand c'est comme ça, c'est que l'enfant veut manger aussi. Et on commence à donner ce qu'on mange à l'enfant. Aussi, quand il mange, il ne tombe pas malade et il grossit ».

La consommation des aliments solides chez l'enfant allaité est en rapport avec sa prise de poids.

\section{1-3.1 La période de sevrage}

Selon la communauté étudiée, le sevrage est l'arrêt de l'allaitement maternel au profit des aliments solides. En général, dans cette communauté, les enfants en état d'allaitement sont sevrés, soit à l'âge de 1 an 6 mois, soit à 2 ans ou plus. En ce qui concerne la période de 1 an 6 mois, elle est plus souvent pratiquée par les jeunes mères. Celles-ci sèvrent aussitôt les enfants en état d'allaitement dans l'optique de garder la fermeté de leurs seins. Selon elles, allaiter un enfant au-delà de 1 an 6 mois, concourt à l'affaissement des seins. Or, les seins affaissés leur conféreraient une apparence de femmes d'âge avancé. Ces pratiques de ces jeunes mères ont des conséquences sur l'état de santé de l'enfant. En effet, celles-ci seraient à l'origine de certains problèmes de santé, tels que la diarrhée, la perte de poids. Quant aux femmes qui ont plus de trois enfants, avec l'expérience, elles allaitent les enfants jusqu'à deux (2) ans, voire plus pour certaines, avant qu'ils ne soient sevrés. Ces femmes estiment que cette façon de nourrir l'enfant allaité le met à l'abri des maladies (anémie, malformations) et il prend de l'embonpoint. À cet âge, l'enfant allaité s'exprime par le parler quand il a faim. Comme le dit Motran 76 ans :

"Les jeunes filles de maintenant, ne veulent pas surveiller enfant, elles ne veulent pas que leurs seins tombent (perdre sa fermeté). Pour cela, elles sèvrent vite (précocement) les enfants, quand c'est comme ça l'enfant tombe régulièrement malade. Sinon, nous on leur demande de nourrir l'enfant au sein jusqu'à deux ans. A deux ans, lorsque l'enfant a faim, il est capable de dire à sa maman. Quand l'enfant est allaité durant deux ans, il ne tombe pas malade n'importe comment, aussi il évolue vite».

Le respect de ces normes sociales assure un bon état de santé de l'enfant allaité. Cette connaissance est transmise aux jeunes mères en vue 
d'améliorer la santé de leurs enfants, et cela renforce encore les liens entre l'enfant allaité et sa mère. Outre, le fait que l'alimentation donne la force et la santé à l'enfant allaité, elle peut aussi dégrader sa croissance, lorsqu'elle n'est pas suivie selon les normes communautaires. Ces normes communautaires qui stipulent qu'un enfant devrait être allaité jusqu'à deux ans.

La prise en charge alimentaire de l'enfant allaité implique également les comportements de la mère.

\section{2- Les comportements de la mère dans la prise en charge de l'enfant allaité}

Pour une bonne santé de l'enfant allaité, la mère observe des restrictions d'ordre alimentaire. La mère se nourrit en fonction des normes de la communauté. Dans la communauté, il y a des aliments que la nourrice s'abstient de consommer pendant la période d'allaitement. Ce sont: la mangue, la sauce arachide ou graine, le silure, le foutou couché, l'œuf, le maïs, l'attiéké. La consommation de ces aliments par la nourrice aurait des conséquences négatives sur l'état de santé de l'enfant allaité.

Tableau 1. Les problèmes de santé de l'enfant allaité suite aux aliments consommés par la mère

\begin{tabular}{|c|c|c|c|c|}
\hline $\begin{array}{c}\text { Nom en langue } \\
\text { française }\end{array}$ & $\begin{array}{c}\text { Nom en } \\
\text { langue locale }\end{array}$ & $\begin{array}{c}\text { Problèmes de } \\
\text { santé suscités }\end{array}$ & \multicolumn{2}{|c|}{$\begin{array}{c}\text { Effectifs des enquêtées sur les } \\
\text { problèmes de santé }\end{array}$} \\
\cline { 5 - 5 } & & Matrones & $\begin{array}{c}\text { Femmes de } \\
\text { troisième âge }\end{array}$ \\
\hline Mangues & Amango & Diarrhée & $10 / 12$ & $4 / 4$ \\
\hline $\begin{array}{c}\text { Sauce graine ou } \\
\text { sauce arachide }\end{array}$ & $\begin{array}{c}\text { Ahé tro ou } \\
\text { ngatè tro }\end{array}$ & $\begin{array}{c}\text { Mal de ventre } \\
\text { ou toux }\end{array}$ & $8 / 12$ & $4 / 4$ \\
\hline Silure & Djuéblé & $\begin{array}{c}\text { Maladies } \\
\text { incurables }\end{array}$ & $11 / 12$ & $4 / 4$ \\
\hline Foutou couché & aliè vouin & Mal de ventre & $7 / 12$ & $4 / 4$ \\
\hline Euf ou maïs & clé n'zoua ou \\
frais & $\begin{array}{c}\text { Ballonnement } \\
\text { de ventre }\end{array}$ & $10 / 12$ & $4 / 4$ \\
\hline Attiéké & atchènin & Constipation & $6 / 12$ & $4 / 4$ \\
\hline
\end{tabular}

Source : Données de terrain 2016

Au regard du tableau, plusieurs problèmes de santé sont causés par la consommation chez la nourrice d'un certain nombre d'aliments. En effet, la consommation par la nourrice de la mangue, la sauce graine ou arachide, le silure, le foutou couché, l'œuf ou maïs frais et l'attiéké entraineraient respectivement les maladies que sont la diarrhée, le mal de ventre ou la toux, les maladies incurables, le mal de ventre, le ballonnement de ventre et la constipation chez l'enfant allaité. Dans l'ensemble, ces résultats sont attestés par $100 \%$ des focus groups réalisés auprès les femmes du troisième âge et par la majorité des matrones interviewées individuellement. 


\section{Discussion}

Il existe bien d'écrits sur la question de la santé infantile. La plupart de ces écrits s'articulent autour de : soins préventifs, restriction alimentaire pour un lait maternel de qualité, restriction sociale pour un lait maternel de qualité, lait maternel comme thérapie chez la nourrice et l'enfant et intérêt de l'aliment de complément

\section{$\checkmark$ Soins préventifs}

Pour préserver l'enfant allaité de la diarrhée lorsque la mère consomme la mangue ; elle fait usage de l'écorce ou des feuilles de manguier pour faire un lavement à l'enfant. Selon Guilhem et Boëtsch (2017), avant la première mise au sein, pour protéger le nouveau-né des impuretés utérines accumulées pendant la période de gestation et lui donner un esprit vif, remuant et agile qu'une chèvre, l'une des parentes de la lignée paternelle réalise sur le nouveauné le rite musulman du «toxantal ». "Ce rite consiste à lui administrer dans la bouche trois gouttes de lait de chèvre mélangées à de l'eau, dans eau ont été préalablement dilués des versets coraniques écrits sur un morceau de papier. Il place l'enfant, être vulnérable, sous la protection d'Allah en l'affiliant à la communauté musulmane. L'utilisation du lait de chèvre obéit à une intentionnalité prophylactique et propitiatoire ».

Cette littérature est en partie similaire aux résultats de cette étude. Guilhem et Boëtsch (2017), montrent que l'allaitement maternel se prépare pour éviter que le nouveau-né soit victime d'un problème de santé et cela se fait à titre préventif. S'inscrivant dans la même veine que ces auteurs, notre étude montre dans la prévention des maladies chez l'enfant allaité, l'abstention de la nourrice à la consommation de la mangue permet de prévenir la diarrhée.

\section{$\checkmark$ Restriction alimentaire pour un lait maternel de qualité}

Pour ce qui est de l'alimentation, Ravaoarisoa et al. (2018) indiquent que chez les Betsiléo à Madagascar, des femmes allaitantes évitent de consommer certains aliments au risque de détériorer la qualité du lait maternel. Ces femmes allaitantes ne mangent pas, « les tubercules, les feuilles vertes et les légumineuses ou la réduction de leur consommation pendant les premiers mois d'allaitement ».

$\mathrm{Au}$ sein de la communauté Baoulé, la consommation de certains aliments par la mère pourrait imprégner le lait maternel et le rendre pathogène. Il s'agit de la mangue, la sauce arachide ou graine, le silure, le foutou couché, l'œuf, le maïs frais, l'attiéké. La consommation desdits aliments par la nourrice peut entrainer chez l'enfant allaité maternellement des problèmes de santé à savoir : la diarrhée, la constipation. En évoquant les tubercules et légumineuses au nombre des restrictions alimentaires, cette étude est en partie similaires à celle de Ravaoarisoa et al. (2018). Cependant, il existe des 
particularités à chaque communauté. Tandis que chez les Baoulé ont parlé de mangue, de silure, du foutou couché, de l'œuf, du maïs frais, les Betsiléo ont souligné le cas des feuilles vertes.

Dans ces deux communautés, Baoulé et Betsiléo l'alimentation de la nourrice est déterminante pour une bonne santé de l'enfant allaité. Les nourrices de chaque communauté observent des interdits d'ordre alimentaire pour contribuer à une meilleure santé de leurs enfants allaités. Le lait maternel est la nourriture fondamentale de l'enfant allaité. Lorsque le lait maternel est trouble du fait du mode alimentaire de la mère, il devient nuisible et peut entrainer des problèmes de santé chez l'enfant allaité. Plusieurs auteurs ayant travaillé sur cette question n'en disent pas moins. Liu et al. (2014) mentionnent que, «la consommation des aliments froids est déconseillée pendant l'allaitement car ils sont considérés comme non favorable à la santé des femmes allaitantes et affectent négativement la quantité et la qualité de la production de lait ». Selon Aid (2015), Cofa (2015), des substances toxiques et autres substances indésirables peuvent également passer dans l'organisme de l'enfant par le biais du lait maternel. Avec certaines mesures de précaution, les femmes qui allaitent peuvent réduire de manière ciblée les risques liés par exemple à la nicotine, à l'alcool ou aux médicaments.

\section{$\checkmark$ Restriction sociale pour un lait maternel de qualité}

Kouamé et Abé (2017), soulignent que les rapports extraconjugaux ont des inconvénients sur l'état de santé de l'enfant. Par exemple, la sexualité extraconjugale. On parle de sexualité extraconjugale lorsque la nourrice a des rapports sexuels en dehors de son conjoint. Cet acte est nommé «kaktchuê » qui signifie « impureté » ou « saleté ». Selon les enquêtées, cet état de fait à des impacts négatifs sur la santé de l'enfant. L'état morbide se caractérise par la morphologie chétive parce que le fait d'avoir les rapports extraconjugaux transforme le lait maternel, lui en enlevant sa qualité nutritive.

Michel et Philippe (n.d), indiquent que le lait devient dangereux pour l'enfant lorsque la mère est confrontée à un problème de santé délicat ou contracte une grossesse. «L'allaitement est arrêté en cas de maladie grave de la mère, et lorsque la femme est à nouveau enceinte ou croit l'être ; en effet, dans ce cas, le lait maternel est considéré comme "mauvais" et pouvant provoquer une maladie diarrhéique réputée dangereuse pour l'enfant allaité », écrivent-ils. Ces auteurs abondent ainsi dans le même que les résultats de l'étude menée. En effet, le lait maternel considéré comme la nourriture principale de l'enfant allaité dans les communautés étudiées, sous l'effet des rayons du soleil et/ou des rapports sexuels, ce lait provoque la diarrhée chez l'enfant allaité. Le lait maternel devient néfaste pour la santé de l'enfant allaité lorsque la mère transgresse des interdits ou des normes sociales. En dehors de la diarrhée évoquée comme pathologie, la présente étude relève que le lait 
maternel pourrait rendre l'enfant allaité de sexe masculin infertile, lorsqu'il tombe sur son sexe.

\section{$\checkmark$ Lait maternel comme thérapie chez la nourrice et l'enfant}

Excepté les problèmes de santé, le lait maternel a des vertus pour une meilleure santé de l'enfant allaité et la mère. Les auteurs comme, Dominique (2010), Green (2019), Koletzko et al. (2016), Bauge (2013), Imorou (2015), INSERM (2014), Henderson et al. (2011) et Jaquet (2011) le montrent dans les travaux respectifs.

Dominique (2010) souligne que «l'allaitement a également des effets bénéfiques pour la santé de la mère. Il est associé à une diminution de l'incidence du cancer du sein avant la ménopause et du cancer de l'ovaire, du diabète de type 2 et de dépression du post-partum. Il est également associé à une réduction du risque de pathologies métaboliques et cardio-vasculaires ». Green (2019), montre les biens faits du lait maternel chez le nourrisson comme chez la mère. Selon elle, « le lait maternel est facile à digérer et offre la bonne quantité de nutriments, car il s'adapte aux besoins des nourrissons à mesure qu'ils grandissent. L'allaitement améliore le développement cognitif et peut protéger contre les infections gastro-intestinales, l'otite moyenne aiguë et les infections des voies respiratoires. (...) L'allaitement est associé à un retard du retour de l'ovulation et à une perte de poids plus importante après l'accouchement, ainsi qu'à une baisse du risque d'hypertension, de diabète, d'hyperlipidémie et de maladie cardiovasculaire. L'allaitement est économique pour les familles il n'est pas nécessaire d'acheter des biberons ni des substituts du lait maternel». Koletzko et al. (2016) signifient également les avantages du lait maternel chez le nourrisson et chez la mère. Pour ceuxci, « la composition nutritionnelle du lait maternel est adaptée de manière optimale aux besoins du nourrisson. Elle évolue au fil de la tétée, mais aussi à mesure que le nourrisson grandit. Le lait maternel des premiers jours (le colostrum) est particulièrement riche en protéines, en anticorps et en facteurs de croissance. Ensuite, il évolue en permanence, toujours en fonction des besoins nutritionnels de l'enfant et de ses capacités digestives. (...) Les femmes qui allaitent ont souvent plus de facilité à perdre du poids après la grossesse. Les réserves de graisse accumulées pendant la grossesse servent de source d'énergie pour la production de lait maternel ». Pascal (2013), indique que l'un des effets collatéraux de l'allaitement est l'attachement et l'interaction plus forte mère-enfant. Or selon lui, on sait que l'attachement augmenté favorise le développement cognitif puisqu'il est lié à plus d'expérience, donc une multiplication des interconnexions neuronales». Imorou (2015), souligne la protection du nouveau-né par le lait maternel. Pour ce dernier, «au nombre des connaissances profanes largement partagées figure l'idée que le lait est un aliment qui permet au nouveau-né de bien se 
porter. Le lait maternel est en général perçu comme un stimulant important de l'intelligence de l'enfant. Il est présenté comme un aliment qui permet d'éviter de nombreuses maladies à l'enfant ». Quant à l'INSERM (2014), on note que les enfants allaités accepteraient de manger plus de fruits et légumes entre 2 et 7 ans. Perçu comme naturel, le lait est plus pratique, plus adapté et sûr (Henderson et al. 2011) relèvent que l'allaitement perçu comme naturel. Jaquet (2011), estime que le lait renforce les liens affectifs entre l'enfant et sa mère. Il offre de multiples expériences sensorielles, car le goût du lait maternel est influencé par l'alimentation de la maman. Il favorise le bon développement de la musculature faciale, buccale et de la mâchoire. (...) Il diminue le risque de développer un cancer du sein avant la ménopause. Il est peu coûteux, écologique et pratique, car le lait est disponible partout, en tout temps et à bonne température.

À l'image de ces travaux, cette étude souligne l'importance du lait maternel pour le développement, la croissance et l'embonpoint de l'enfant allaité. Le lait maternel consolide les liens entre la mère et son enfant, réduit les pleurs de l'enfant, c'est le moment de l'allaitement la mère parle beaucoup à son enfant, il protège l'enfant contre plusieurs maladies.

\section{$\checkmark$ Intérêt de l'aliment de complément}

Il existe divers types de compléments et ceux-ci sont donnés précocement aux enfants en état d'allaitement. Djadou et al. (2017), indiquent dans leur étude à Tchaoudjo (Togo) que l'eau et la bouillie sont des aliments qui sont donnés aussitôt aux enfants allaités. Selon les résultats de leur étude, «l'eau et la bouillie $(63,5 \%)$ étaient les aliments les plus introduits par les mères en plus du lait maternel avant 6 mois. L'eau de puits était la plus donnée $(60,6 \%)$. Parmi les mères ayant introduit des aliments en plus du lait maternel avant six mois, $69,9 \%$ avaient donné de la bouillie locale à base de mil ou de maïs contre 30,1\% de bouillie de céréale enrichie ». De même Sibetcheu et al. (2017), montrent qu'au Cameroun, les mères introduisent très tôt l'aliment de complément. Ils montrent que des liquides autre le lait maternel et des aliments solides ou semi-solides sont introduits très tôt, avant 6 mois dans l'alimentation de l'enfant allaité. Ainsi, selon leurs résultats, chez les enfants de moins de 2 mois, $7 \%$ avaient reçu d'autres liquides et $8 \%$ avaient reçu d'autres laits ou des produits laitiers (fromage et yaourt). Pour la tranche 2-3 mois, $19 \%$ avaient déjà reçu des aliments solides à base de céréales et pour la tranche 4-5 mois, $9 \%$ avaient consommé des aliments solides à base de poissons, volailles, viandes ou œufs. À 6-9 mois, seulement $30 \%$ des enfants reçoivent des aliments riches en vitamine A ». Gray et al. (2014) dans une étude réalisée au Congo, notent que pour éviter les pleurs des enfants allaités, ils sont nourris tôt avec les aliments de complément. Ce propos rapporté par cet auteur en dit mieux : 
"Je sais qu'il faut allaiter mon enfant jusqu'à 6 mois. J'ai essayé. Pourtant il pleurait tout le temps; il me fatiguait. J'ai commencé par ajouter des biberons supplémentaires par intervalle de ses tétées lorsqu'il a eu 2 mois. Puis, à 3 mois, j'ai commencé à alourdir ses biberons avec des cuillères de farines infantiles. A quatre mois, il prenait déjà une bouillie de céréales précuite. A 5 mois il était initié au plat familial. ». " Je ne dormais plus la nuit. Mon bébé réclamait de téter tout le temps. Plus il grandissait, plus il réclamait. Peu avant qu'il atteigne 3 mois, j'ai décidé de lui donner une bouillie à base de farine industrielle qu'on trouve sur le marché. Le jour où j'ai commencé, j'ai dormi toute la nuit sans qu'il pleure pour réclamer de téter. J'ai donc résolu de continuer»

Selon Salanave et al. (2012), l'étude Epifane réalisée en France montre qu'à un mois les nourrissons n'étaient plus que $35 \%$ à être allaités en exclusif et $54 \%$ en mixte. Par ailleurs, HKI (2010), soutient que l'introduction précoce d'aliments non adaptés aux différentes étapes de la croissance du jeune enfant prédispose ce dernier à la malnutrition avec des répercussions psychophysiologiques sur son devenir d'adulte.

Cette étude réalisée en Côte d'Ivoire témoigne de l'interculturalité de cette pratique qui consiste à donner des aliments de complément aux enfants allaités. Tout comme au Togo, au Cameroun, au Congo et en France, les bouillies et les plats familiaux sont donnés précocement à l'enfant allaité, afin qu'il puisse dormir et la mère profite de ces moments pour faire ces activités quotidiennes en Côte d'Ivoire, précisément chez les communautés baoulé Faly de Bouaké et Nananfouè de Yamoussoukro.

\section{Conclusion}

Abordant la problématique de la prise en charge nutritionnelle de l'enfant allaité, cet article a permis de comprendre comment celle-ci est faite dans les communautés Baoulé (Faly et Nananfouè) en Côte d'Ivoire. Parti du constat que les enfants allaités sont précocement nourris avec des aliments divers et que la nourrice est soumise à des restrictions alimentaires pendant cette prise en charge, l'étude relève deux faits majeurs. Dans un premier temps, la prise en charge nutritionnelle de l'enfant allaité, en communauté Faly et Nananfouè, repose sur une alimentation mixte, intégrant l'allaitement maternel et les aliments de complément. Comme aliments de complément, les bouillies de mil, riz, maïs et des plats tels que le placali sont donnés à l'enfant allaité dans ces communautés. Dans un second temps, l'étude montre que $100 \%$ des focus groups réalisés auprès les femmes du troisième âge et par la majorité des matrones interviewées individuellement attestent que la 
consommation par la nourrice de la mangue, la sauce graine ou arachide, le silure, le foutou couché, l'œuf ou maïs frais et l'attiéké entraineraient respectivement les maladies que sont la diarrhée, le mal de ventre ou la toux, les maladies incurables, le mal de ventre, le ballonnement de ventre et la constipation chez l'enfant allaité. Dans la nécessaire collaboration entre sciences biomédicales et sciences sociales notamment l'anthropologie et la sociologie, la prise en compte des pratiques communautaires en matière de prise en charge nutritionnelle des enfants allaité, pourrait servir de catalyseur aux professionnels de la santé (sages-femmes, pédiatres surtout) pour une communication pour le changement de comportements plus efficace.

\section{References :}

1. Aid (2015). aid infodienst e.V. Multiplikatorenfortbildung Primäre Allergieprävention.

2. Bauge, P. (2013). Développement cérébral et cognitif de l'enfant : impact de l'allaitement maternel, https://lemondeetnous.cafesciences.org/2013/03/developpement-cerebral-et-cognitif-de-lenfantimpact-de-lallaitement-maternel/

3. Caraël, M. \& Msellati, P. (n.d). Représentation et pratiques de l'allaitement à Kigali, Rwanda,

pp.4.http://horizon.documentation.ird.fr/exlhttp://horizon.documentat ion.ird.fr/exldoc/pleins_textes/pleins_textes_7/b_fdi_03_02/0100247 42

4. COFA (2015). Commission fédérale de l'alimentation COFA : Alimentation durant les 1000 premiers jours de vie de la conception au 3e anniversaire : Rapport de la COFA. Zurich : Office fédéral de la sécurité alimentaire et des affaires vétérinaires.t Ali Collection Expertise collective, Inserm, Paris.

5. Djadou et al. (2017). Évaluation de l'allaitement maternel exclusif chez les enfants de 0 à six mois dans le district de Tchaoudjo (Togo), J Afr Pediatr Genet Med, pp.2.

6. Gray et al. (2014). Perception de l'allaitement maternel et de la diversification alimentaire dans une zone urbaine congolaise. The Pan African Medical Journal.

7. Green, M. (2019). Inf. aut., M. Sc. inf., IBCLC Comité canadien pour l'allaitement, Vancouver (Colombie-Britannique). L'allaitement maternel. https://www.canada.ca/fr/santepublique/services/publications/vie-saine/soins-meres-nouveau-nelignes-directrices-nationales-chapitre-6.html

8. Guilhem, D. \& Boëtsch, G. (2017). Lait maternel ou lait de vache ? La production du corps de l'enfant par deux liquides nourriciers chez les Peuls du Ferlo (Sénégal), in Arena F., Foehr-Janssens Y., 
Papaikonomou I. \& Prescendi F. (éds), Allaitement entre humains et animaux : représentations et pratiques de l'Antiquité à aujourd'hui. Anthropozoologica 52 (1) : 91-101.

https://doi.org/10.5252/az2017n1a8

9. Helen Keller International-Cote d'ivoire (HKI-CI) (2010). Recherche formative sur les pratiques d'alimentation du nourrisson et du jeune enfant (REFACE) PACE/IYCN, Rapport final, Abidjan, Côte d'Ivoire, Helen Keller International-Côte d'Ivoire, 86 p.

10. Henderson et al. (2011). Men and infant feeding: perceptions of embarrassment, sexuality, and social conduct in white low-income british men. Birth, p. 61-70.

11. Imorou, A-B. (2015). «à chacun son âme » : les raisons de la difficile quantification des comportements dans l'allaitement maternel exclusif au bénin. Rev. ivoir. anthropol. sociol. KASA BYA KASA, $\mathrm{n}^{\circ} 29$, () EDUCI 2015.

12. INSERM (2014). Inégalités sociales de santé en lien avec l'alimentation et l'activité physique.

13. Jaquet, M. (2011). Mère et enfant. 3éme édition actualisée. Société Suisse de Nutrition SSN. Berne.

14. Koletzko et al. (2016). Ernährung und Bewegung von Säuglingen und stillenden Frauen. Monatszeitschrift Kinderheilkunde Sonderdruck.

15. Kouamé, A.M.N \& Abé, N.N.(2017). Perception sociale de la malnutrition dans la communauté akye bofanmun en Côte D'ivoire. International Journal of Multidisciplinary Research and Development.

16. Liu, Y. Q., Petrini, M., \& Maloni, J. A. (2014). "Doing the month": postpartum practices in Chinese women. Nurs Health Sci.

17. Lupton, D. (2013). Infant embodiment and interembodiment: A review of sociocultural perspectives, Childhood, p 37-50. Online ISSN: 23494182, Print ISSN: 2349-5979, Impact Factor: RJIF 5.72 Volume 4, Page No. 105-110.

18. Ravaoarisoa1 et al. (2018). Habitude alimentaire des mères pendant la grossesse et l'allaitement, région Amoron'i Mania Madagascar : étude qualitative, in Pan African Medical Journal.

19. Reckwitz, A. (2002). Toward a theory of social practices: A development in culturalist theorizing, European Journal of Social Theory, vol. 5, $\mathrm{n}^{\circ}$ 2, DOI : 10.1177/13684310222225432.

20. Salanave, B., De Launay, C., Guerrisi, C., \& Castetbon, K. (2012). Taux d'allaitement maternel à la maternité et au premier mois de l'enfant. Résultats de l'étude Epifane, France. BEH 2012, p. 383-387.

21. Sibetcheu et al. (2017). Allaitement maternel, état nutritionnel des enfants et des femmes.

22. Turck, D. (2010). Plan d'action : Allaitement maternel, p.7. 